\title{
Selection of Candidates for Endovascular Treatment: Characteristics According to Three Different Selection Methods
}

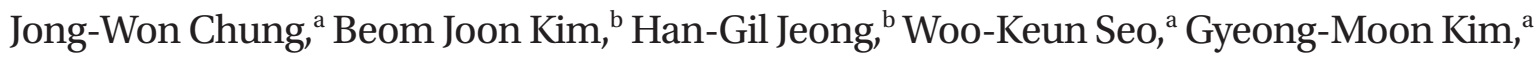 \\ Cheolkyu Jung, ${ }^{\mathrm{c}}$ Moon-Ku Han, ${ }^{\mathrm{b}}$ Hee-Joon Bae, ${ }^{\mathrm{b}}$ Oh Young Bang ${ }^{\mathrm{a}}$ \\ ${ }^{a}$ Department of Neurology, Samsung Medical Center, Sungkyunkwan University School of Medicine, Seoul, Korea \\ ${ }^{b}$ Department of Neurology, Seoul National University Bundang Hospital, Seoul National University College of Medicine, Seongnam, Korea \\ 'Department of Radiology, Seoul National University Bundang Hospital, Seoul National University College of Medicine, Seongnam, Korea
}

Background and Purpose To investigate the number and characteristics of patients eligible for endovascular treatment (EVT) determined using three different selection methods: clinical-core mismatch, target mismatch, and collateral status.

Methods Using the data of consecutive patients from two prospectively maintained registries of university medical centers, the number and characteristics of patients according to the three selection methods were investigated and their correlation was analyzed. Patients with anterior circulation stroke due to occlusion of the middle cerebral and/or internal carotid artery and a National Institute of Health Stroke Scale (NIHSS) score of $\geq 6$ points, who arrived within 8 hours or between 6 and 12 hours of symptom onset and underwent magnetic resonance imaging prior to EVT, were included. Collateral status was assessed using magnetic resonance perfusion-derived collateral flow maps.

Results Three hundred thirty-five patients were investigated; the proportions of patients who were eligible and ineligible for EVT in all three selection methods were both small $(n=85,25.4 \%$; $n=54$, $16.1 \%$, respectively). The intercorrelation among the three selection methods was low ( $k=0.235)$. The baseline NIHSS score and onset-to-selection time interval were associated with the presence of clinical-core mismatch, while the penumbra/core volume ratio and onset-to-selection time interval were related to target mismatch; none of these variables were associated with collateral status. The infarct core volume was associated with favorable profiles in all three selection methods.

Conclusions Although the application of individual selection methods resulted in favorable outcomes after EVT in clinical trials, there is a significant discrepancy in EVT eligibility depending on the selection method used.

Keywords Endovascular treatment; Selection; Mismatch; Collateral
Correspondence: Oh Young Bang Department of Neurology, Samsung Medical Center, Sungkyunkwan University School of Medicine, 81 Irwonro, Gangnam-gu, Seoul 06351, Korea Tel: +82-2-3410-3599

Fax: +82-2-3410-0052

E-mail: ohyoung.bang@samsung.com

Received: June 20, 2019 Revised: August 30, 2019 Accepted: September 3, 2019 


\section{Introduction}

The beneficial effect of endovascular treatment (EVT) with mechanical thrombectomy has been confirmed in acute ischemic stroke (AIS) in pivotal randomized controlled trials (RCTs). A meta-analysis of individual patient data from five RCTs showed that the benefit of EVT decreased with the delay in recanalization after stroke onset. ${ }^{1}$ However, recent phase III RCTs on EVT in an extended time window reported considerable and remarkable functional recovery after EVT compared with that after medical treatment in carefully selected patients., ${ }^{2,3} \mathrm{EVT}$ was initiated between 6 and 16 hours after symptom onset in patients with target mismatch in the Diffusion and Perfusion Imaging Evaluation for Understanding Stroke Evolution (DEFUSE)-3 trial ${ }^{3}$ and between 6 and 24 hours after symptom onset in patients with mismatch between clinical presentation and ischemic core, as measured using diffusion-weighted imaging (DWI)/computed tomography (CT) perfusion in the DWI or CT Perfusion Assessment with Clinical Mismatch in the Triage of Wake Up and Late Presenting Strokes Undergoing Neurointervention with Trevo (DAWN) trial. ${ }^{2}$ Retrospective studies showed that good collaterals predict better clinical responses to EVT beyond 5 hours from onset. ${ }^{4-7}$ Prospective studies on EVT based on the collateral status assessed on CT or magnetic resonance imaging (MRI) are ongoing (https://clinicaltrials.gov/ ct2/show/NCT03234634 and https://clinicaltrials.gov/ct2/ show/NCT02668627).

Different selection methods use varying parameters to select candidate patients for EVT, and it is possible that patients who are eligible for EVT in one selection method are considered ineligible in other selection methods, which may lead to controversies in patient treatment decisions in clinical practice. We hypothesized that the number of patients with AIS who are eligible for EVT would differ depending on the selection method used. Thus, we applied three selection methods (clinical-core mismatch, target mismatch, and collateral status) in two separate cohorts of AIS due to large-vessel occlusion. We investigated the number of patients eligible for EVT and compared their characteristics depending on the selection method used.

\section{Methods}

\section{Patient selection}

Using the data of consecutive patients from two prospectively maintained registries of university medical centers (Samsung Medical Center [SMC], Seoul and Seoul National University Bundang Hospital [SNUBH], Seongnam), we identified patients who (1) had a National Institute of Health Stroke Scale (NIHSS) score of $\geq 6$ points at admission, (2) had a prestroke modified Rankin Scale score of 0 or 1 point, (3) had an acute infarction within the middle cerebral artery (MCA) territory due to occlusion of the internal carotid artery and/or proximal MCA (M1 segment), and (4) underwent pretreatment brain MRI, including DWI, magnetic resonance perfusion (MRP), and magnetic resonance angiography (MRA).

To apply the three selection methods to different settings in terms of the time to selection, patients with clear symptom onset time who presented at SMC within 8 hours of symptom onset (from July 2005 to January 2016) and those who presented at SNUBH within 6 to 12 hours of symptom onset (from January 2011 to September 2016) were evaluated. The two time periods were selected on the basis of available patient data with sufficient clinical and MRI parameters for the selection classification in each center. According to the institutional hyperacute stroke critical pathway in SNUBH, patients who arrived within 6 hours of symptom onset underwent CT for EVT candidate selection. This study was approved by the local Institutional Review Boards of the participating centers, with the requirement of patient consent waived owing to the anonymity of the collected data and the minimal risk to the participants.

The patients were evaluated on the basis of their demographic data, medical history, vascular risk factors, routine blood test findings (total cholesterol, triglyceride, high-density and low-density lipoprotein cholesterol, and C-reactive protein levels), brain imaging findings (multimodal MRI and vascular studies), and cardiological assessment findings (electrocardiography, $\geq 24$-hour cardiac telemetry, or echocardiography) according to the study protocol.

\section{Three selection methods}

The three selection methods were previously described elsewhere and are shown in Table 1., ${ }^{2,3}$,

(1) Clinical-core mismatch was diagnosed as described in the DAWN trial based on the following factors: ${ }^{2}$ age $\geq 80$ years, $\mathrm{NI}-$ HSS score $\geq 10$ points, and infarct volume $<21 \mathrm{~mL}$; age $<80$ years, NIHSS score $\geq 10$ points, and infarct volume $<31 \mathrm{~mL}$; and age $<80$ years, NIHSS score $\geq 20$ points, and infarct volume of 31 to $51 \mathrm{~mL}$.

(2) Target mismatch was diagnosed as described in the DEFUSE-3 trial based on the following factors: ${ }^{3}$ infarct volume on DWI $<70 \mathrm{~mL}$, ischemic tissue volume/initial infarct volume ratio $\geq 1.8$, and absolute volume of potentially reversible ischemia ( max,$>6$ seconds) $\geq 15 \mathrm{~mL}$. MRP post-processing and data analysis were performed as described in our previous study. ${ }^{9}$ MRI volume measurements were semi-automatically performed using a computer-assisted volumetric analysis program 
Table 1. Differences among the three selection methods for endovascular treatment of acute ischemic stroke

\begin{tabular}{|c|c|c|c|}
\hline & Clinical-core mismatch & Target mismatch & Collateral status \\
\hline Parameters & $\begin{array}{l}\text { Age } \\
\text { NIHSS score } \\
\text { Core size }\end{array}$ & $\begin{array}{l}\text { Core } \\
\text { Penumbrae }\end{array}$ & Collateral grade alone \\
\hline Candidate patients & $\begin{array}{l}\text { A. Patients aged } \geq 80 \text { years with an } \\
\text { NIHSS score of } \geq 10 \text { points and in- } \\
\text { farct core volume of }<21 \mathrm{~mL} \\
\text { B. Patients aged }<80 \text { years with an } \\
\text { NIHSS score of } \geq 10 \text { points and in- } \\
\text { farct core volume of }<31 \mathrm{~mL} \text { or with } \\
\text { an NIHSS score of } \geq 20 \text { points and } \\
\text { infarct core volume of }<51 \mathrm{~mL}\end{array}$ & $\begin{array}{l}\text { A. Predicted infarct core volume of } \leq 70 \mathrm{~mL} \\
\text { B. Ratio between the volumes of critically hy- } \\
\text { poperfused tissues (Tmax, }>6 \mathrm{sec} \text { ) } \\
\text { C. Ischemic core of } \geq 1.8 \text {, with an absolute dif- } \\
\text { ference of } \geq 15 \mathrm{~mL}\end{array}$ & $\begin{array}{l}\text { A. Grade 3: slow but complete collateral flow } \\
\text { in the occluded MCA territory. } \\
\text { B. Grade 4: rapid and complete collateral flow } \\
\text { in the occluded MCA territory. }\end{array}$ \\
\hline Key characteristics & $\begin{array}{l}\text { Highly dependent on clinical features } \\
\text { (location of infarcts) }\end{array}$ & Delayed arterial reperfusion (tissue perfusion) & $\begin{array}{l}\text { Delayed reperfusion (retrograde collaterals) } \\
\text { Not considering core }\end{array}$ \\
\hline Advantages & $\begin{array}{l}\text { Simple assessment } \\
\text { No post-processing }\end{array}$ & $\begin{array}{l}\text { Pathophysiologically plausible } \\
\text { Clear visualization }\end{array}$ & Pathophysiologically plausible \\
\hline Disadvantages & $\begin{array}{l}\text { Highly dependent on core size and } \\
\text { location }\end{array}$ & Post-processing (but fast-automated) & Post-processing (but fast-automated) \\
\hline Relevant studies & DAWN trial ${ }^{2}$ & DEFUSE-3 trial $\left.\right|^{3}$ & $\begin{array}{l}4 \text { Retrospective studies } \\
2 \text { Prospective studies (ongoing CoSETS and } \\
\text { FAST-COLL studies) }\end{array}$ \\
\hline
\end{tabular}

NIHSS, National Institute of Health Stroke Scale; DAWN, Diffusion-Weighted Imaging or Computed Tomography Perfusion Assessment with Clinical Mismatch in the Triage of Wake Up and Late Presenting Strokes Undergoing Neurointervention with Trevo; DEFUSE, Diffusion and Perfusion Imaging Evaluation for Understanding Stroke Evolution; CoSETS, Collateral-based reSetting of Endovascular Treatment Time Window for Stroke; FAST-COLL, Fast Analysis SysTem for COLLaterals.

(Medical Image Processing, Analysis and Visualization version 3.0, NIH, Bethesda, MD, USA) by an investigator who was blinded to the clinical information.

(3) Collateral status was assessed using collateral flow maps: ${ }^{8}$ Collateral flow maps were generated on the basis of source data derived from dynamic susceptibility contrast-enhanced MRP (DSC-MRP) and were automatically generated using an in-house software (Fast Analysis SysTem for COLLaterals) developed using MATLAB (MathWorks, Natick, MA, USA), as previously reported. ${ }^{8}$ This MRP-based collateral flow map method was applied in a French multicenter trial. ${ }^{10}$ In this study, the patients were divided into two groups according to their grade: poor (American Society of Interventional and Therapeutic Neuroradiology [ASITN] and the Society of Interventional Radiology [SIR] scale grade 1-2, no collaterals or some defect) or good (grade 3-4, complete collateral flow to the occluded vascular bed).

\section{MRP methods and image analysis}

At SMC, MRI was performed using the $3 \mathrm{~T}$ Philips Achieva magnetic resonance scanner (Philips Medical Systems, Best, the Netherlands). Typical MRI sequences for acute stroke included at least DWI, DSC-MRP, fluid-attenuated inversion recovery, and MRA of the cervical and intracranial vessels (three-dimensional time-offlight MRA and contrast-enhanced MRA, including the extracrani- al carotid and vertebral arteries). DWI was performed with two levels of diffusion sensitization (b values of 0 and $1,000 \mathrm{sec} / \mathrm{mm}^{2}$; slice thickness of 5 to $7 \mathrm{~mm}$; no gap). DSC-MRP was performed using gradient-echo and echo-planar imaging techniques after intravenous administration of gadolinium (Dotarem [gadoterate meglumine], Guerbet, Aulnay-sous-Bois, France), with a repetition time of $1,718 \mathrm{~ms}$ for a total acquisition time of approximately 90 seconds with 20 slices. The contrast agent was injected at a dose of $0.1 \mathrm{mmol} / \mathrm{kg}$, with a flow rate of $3 \mathrm{~mL} / \mathrm{sec}$, using a power injector to the antecubital vein via an 18-gauge intravenous cannula approximately 7 seconds after beginning the acquisition. The other parameters for DSC-MRP were as follows: echo time $=35 \mathrm{~ms}$, flip angle $=40^{\circ}$, acquisition matrix $=128 \times 128$, field of view $=24 \times 24$ $\mathrm{cm}^{2}$, section thickness $=5 \mathrm{~mm}$, and intersection gap $=2 \mathrm{~mm}$. In total, 1,000 DSC-MRP raw images, composed of 50 time points per slice, were obtained.

At SNUBH, MRI was performed using the $1.5 \mathrm{~T}$ or $3 \mathrm{~T}$ magnetic resonance systems (Intera 1.5 T or Intera Achieva or Ingenia $3 \mathrm{~T}$, Philips Health Care). Typical MRI sequences for acute stroke included at least DWI, DSC-MRP, fluid-attenuated inversion recovery before and after contrast agent administration, and MRA of the cervical and intracranial vessels (three-dimensional time-of-flight MRA and contrast-enhanced MRA, including the extracranial carotid and vertebral arteries). DWI was also performed with two levels of diffusion sensitization (b 
values of 0 and $1,000 \mathrm{sec} / \mathrm{mm}^{2}$; slice thickness of $5 \mathrm{~mm}$; interslice gap of 1 to $2 \mathrm{~mm}$ ). DSC-MRP was performed using gradient-echo and echo-planar imaging techniques after intravenous administration of gadolinium (Gadovist, Bayer Inc., Toronto, $\mathrm{ON}$, Canada), with a repetition time of $1,500 \mathrm{~ms}$ for a total acquisition time of approximately 60 seconds with 19 slices. The contrast agent was injected at a dose of $0.1 \mathrm{mmol} / \mathrm{kg}$, with a flow rate of $4 \mathrm{~mL} / \mathrm{sec}$, using a power injector to the antecubital vein via an 18-gauge or a 20-gauge intravenous cannula approximately 7 seconds after beginning the acquisition. The other parameters for DSC-MRP were as follows: echo time $=40$ $m s$, flip angle $=90^{\circ}$, echo train length $=63$, acquisition matrix $=120 \times 120$, field of view $=23 \times 23 \mathrm{~cm}^{2}$, section thickness $=5$ $\mathrm{mm}$, and intersection gap $=6 \mathrm{~mm}$. In total, 760 DSC-MRP raw images, composed of 40 time points per slice, were obtained.

\section{Statistical analysis}

Demographic data, clinical characteristics, and clinical outcomes were compared between the groups. The onset-to-selection time interval was defined as the time interval between symptom onset and MRI completion time. Pearson's chi-square test or Fisher's exact test was used for analyzing categorical variables and Student's t-test or Mann-Whitney $U$ test for analyzing continuous variables. The correlation among the three selection methods was evaluated using Cohen's $\mathrm{K}$ coefficient. All analyses were performed using Stata version 13 (StataCorp, College Stations, TX, USA).

\section{Role of the funding source}

The sponsor of the study had no role in the study design, data analysis, data interpretation, writing of the report, or decision to publish the results. The corresponding author claims final responsibility for the decision to submit for publication.

\section{Data availability statement}

Any data not published within the article will be shared by request from any qualified investigator.

\section{Results}

A total of 232 patients from SMC and 103 patients from SNUBH who met the inclusion criteria were included. The baseline characteristics according to the institution and selection method are presented in Table 2 and Supplementary Table 1.

\section{Proportion of patients eligible for EVT in the three selection methods}

The proportion of the eligible patients according to the selection
Table 2. Patient characteristics in the two cohorts

\begin{tabular}{lll}
\hline Characteristic & \multicolumn{1}{c}{ SMC cohort } & SNUBH cohort \\
\hline No. of cases & $232(69.3)$ & $103(30.7)$ \\
Onset-to-selection time interval (min) & $144(104-210)$ & $441(411-496)$ \\
Age (yr) & $65.0 \pm 14.1$ & $70.6 \pm 11.5$ \\
Female sex & $99(42.7)$ & $42(40.8)$ \\
NIHSS score & $13(9-18)$ & $14(10-19)$ \\
Atrial fibrillation & $86(44.1)$ & $58(56.3)$
\end{tabular}

Values are presented as number (\%), median (interquartile range), or mean \pm standard deviation.

SMC, Samsung Medical Center; SNUBH, Seoul National University Bundang Hospital; NIHSS, National Institutes of Health Stroke Scale.

method is shown in Figure 1. In both the SMC and SNUBH cohorts, the proportion of patients who were eligible for EVT in all three selection methods was small: 63 of the 232 patients (27.2\%) from the SMC cohort and 23 of the 103 patients (22.3\%) from the SNUBH cohort. Similarly, only a small proportion of patients were ineligible for EVT in all three selection methods: 33 of the 232 patients (14.2\%) from the SMC cohort and 21 of the 103 patients (20.4\%) from the SNUBH cohort. In both cohorts, the proportion of patients who were eligible for EVT differed depending on the selection method used: highest in the collateral status selection method, lowest in the clinical-core mismatch selection method, and intermediate in the target mismatch selection method. The remaining 58.6\% of the patients in the SMC cohort and $57.3 \%$ of the patients in the SNUBH cohort were reclassified depending on the selection method used. The intercorrelation among the three selection methods was low (clinical-core mismatch vs. target mismatch, $\mathrm{K}=0.368$; target mismatch vs. collateral status, $\mathrm{K}=0.238$; collateral status vs. clinical-core mismatch, $\mathrm{K}=0.128$; overall [collateral status vs. target mismatch vs. clinical-core mismatch], $\mathrm{k}=0.235$ ). A discrepancy in EVT eligibility according to the selection methods was also demonstrated in a later time period (onset-to-selection time interval, $>6$ hours) among the pooled patients from the two cohorts (Supplementary Figure 1). A typical example is provided in Figure 2.

\section{Differential factors affecting eligibility for EVT among the selection methods}

The clinical and imaging characteristics among the patients considered eligible for EVT in the three selection methods are demonstrated in Table 3. The patients with clinical-core mismatch had a higher initial NIHSS score than those with a positive target mismatch profile and a good collateral status. The initial infarct core volume did not differ among the three selection methods; however, the penumbra/ischemic core ratio was significantly associated with a positive target mismatch 


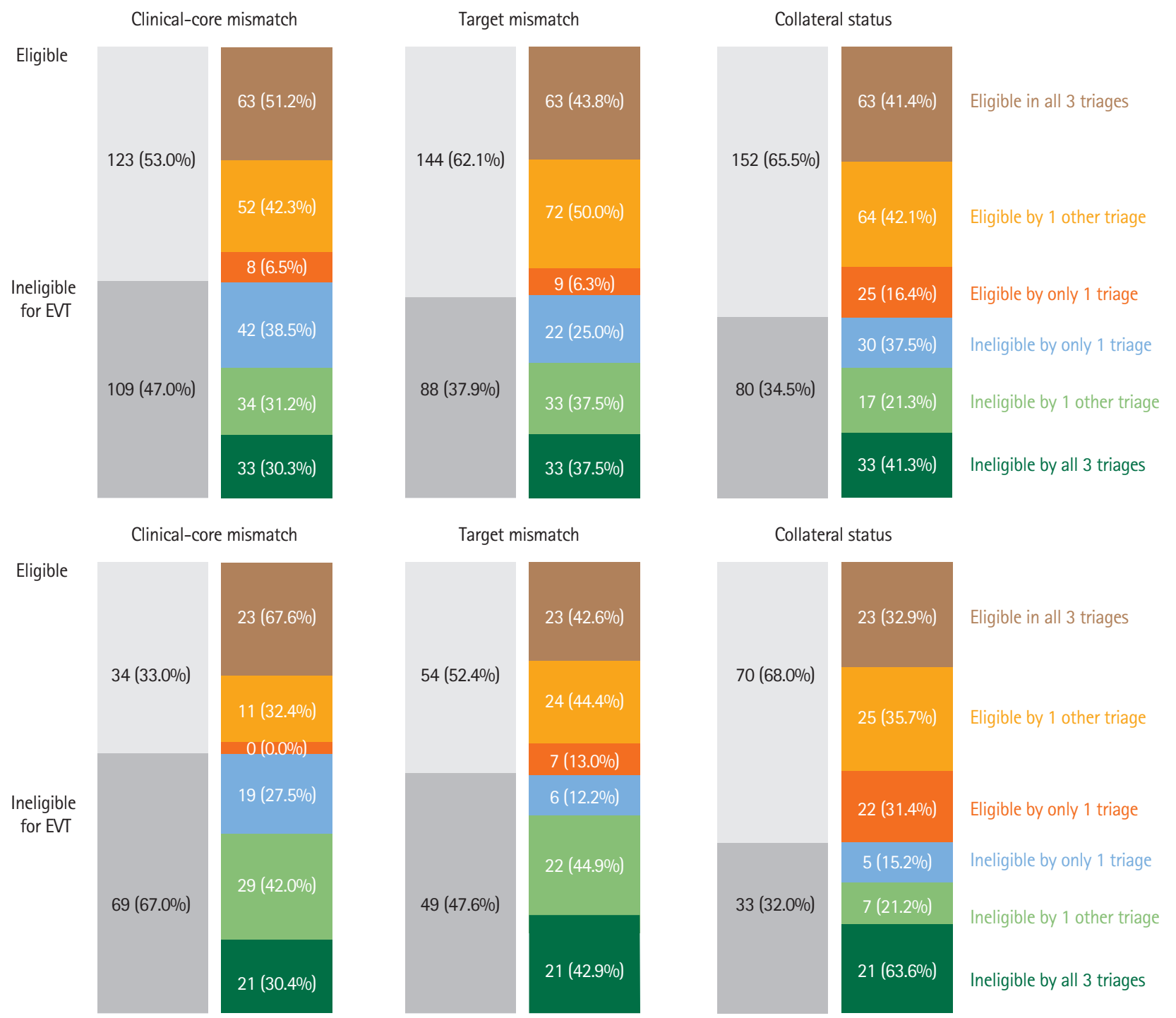

Figure 1. Number of patients with favorable and unfavorable profiles according to the three selection methods. (A) Samsung Medical Center cohort (onsetto-selection time interval, <8 hours), (B) Seoul National University Bundang Hospital cohort (onset-to-selection time interval, 6 to 12 hours). EVT, endovascular treatment.

profile. The onset-to-selection time interval was significantly longer in the patients with a good collateral status.

In the multivariable analyses, the baseline NIHSS score and onset-to-selection time interval were associated with the presence of clinical-core mismatch, while the penumbra/core volume ratio and onset-to-selection time interval were related to target mismatch; none of these variables were associated with collateral status (Table 4). The infarct core volume was associated with favorable profiles in all three selection methods.

\section{Discussion}

The main finding of this study is that the number of patients with AIS who are eligible for EVT differs depending on the se- lection method used (i.e., clinical-core mismatch, target mismatch, and collateral status). This is the first study comparing all three selection methods for EVT in patients with AIS within the MCA territory.

The number of eligible patients and predictive values differed among the three selection methods. Only one of four patients was classified as eligible for EVT and one of five patients as ineligible in all three selection methods. The remaining 58.6\% of the patients in the SMC cohort and 57.3\% of the patients in the SNUBH cohort were reclassified according to other selection methods (either from eligible to ineligible or vice versa).

Certain key factors considered in one selection method did not influence the eligibility for EVT in the other selection methods, suggesting that these selection methods provide differen- 


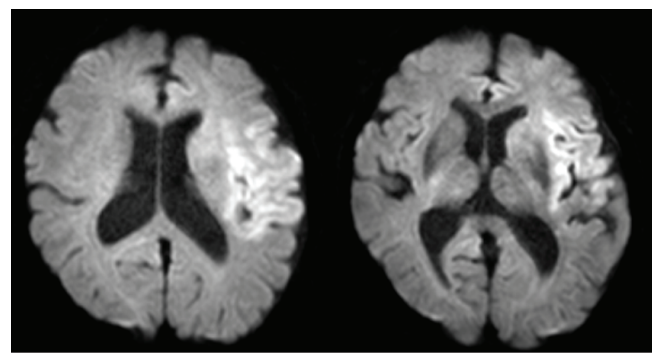

Ischemic core volume: $55 \mathrm{~mL}$

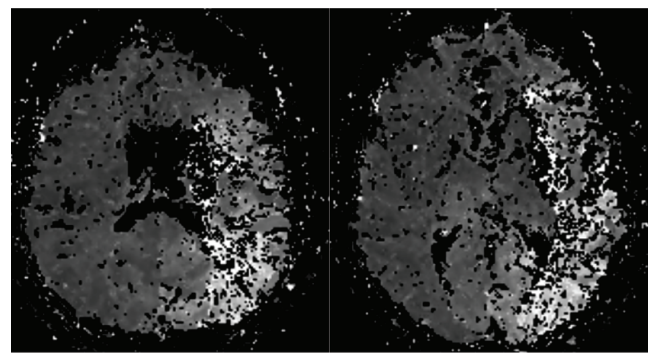

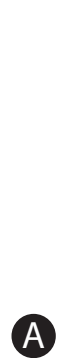

A

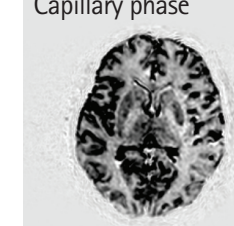

Venous-late venous phase

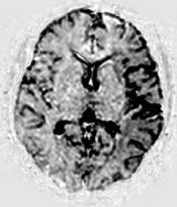

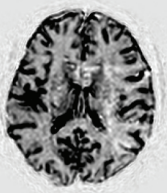
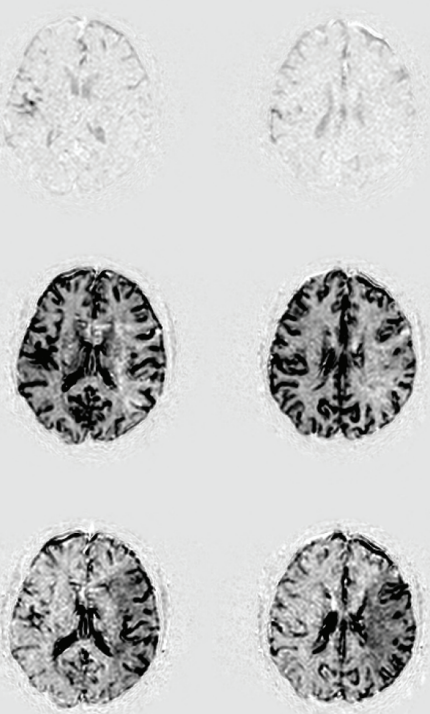

Penumbra volume: $117 \mathrm{~mL}$

B

Figure 2. Typical examples of mismatch among the selection methods. Images of a 76-year-old man with left middle cerebral artery occlusion with an initial National Institutes of Health Stroke Scale score of 21 points and an onset-to-selection time interval of 520 minutes. The patient is classified as ineligible for endovascular treatment in the clinical-core mismatch selection method $(A)$ but eligible in the target mismatch $(A, B)$ and collateral status selection methods $(C)$.

Table 3. Comparisons of the selection components in the patients with favorable profiles

\begin{tabular}{|c|c|c|c|c|}
\hline Variable & Clinical-core mismatch, present & Target mismatch, present & Collateral status, good & $P$ \\
\hline No. of patients & $157(46.9)$ & $198(59.1)$ & $222(66.3)$ & \\
\hline Age $(y r)$ & $66.9 \pm 12.6$ & $66.6 \pm 12.2$ & $66.6 \pm 13.3$ & 0.969 \\
\hline$<80$ & 141 (89.8) & 178 (89.9) & $192(86.5)$ & \\
\hline$\geq 80$ & $16(10.2)$ & $20(10.1)$ & $30(13.5)$ & \\
\hline NIHSS score (point) & $15(12-19)$ & $13(9-18)$ & $12(9-17)$ & $<0.001$ \\
\hline$<10$ & $0(0)$ & $53(26.8)$ & $68(30.6)$ & \\
\hline $10-19$ & $125(79.6)$ & $119(60.1)$ & $127(57.2)$ & \\
\hline$\geq 20$ & $32(20.4)$ & $26(13.1)$ & $27(12.2)$ & \\
\hline Infarct core volume (mL) & $21.8 \pm 27.9$ & $21.2 \pm 21.5$ & $22.8 \pm 30.8$ & 0.820 \\
\hline$<30$ & $135(86.0)$ & $155(78.3)$ & $172(77.5)$ & \\
\hline $30-70$ & $22(14.0)$ & $43(21.7)$ & $36(16.2)$ & \\
\hline$>70$ & $0(0)$ & $0(0)$ & $15(6.8)$ & \\
\hline Penumbra/core volume ratio & $14.5 \pm 57.8$ & $16.5 \pm 55.5$ & $13.9 \pm 52.5$ & 0.881 \\
\hline$\geq 1.8$ & $124(79.0)$ & $198(100.0)$ & $158(71.2)$ & $<0.001$ \\
\hline$<1.8$ & $33(21.0)$ & $0(0)$ & $64(28.2)$ & $<0.001$ \\
\hline Collateral status & & & & $<0.001$ \\
\hline Grade 1, poor & $14(8.9)$ & $15(7.6)$ & $0(0)$ & \\
\hline Grade 2, intermediate & $28(17.8)$ & $33(16.7)$ & $0(0)$ & \\
\hline Grade 3, good & 49 (31.2) & $84(42.4)$ & $105(47.3)$ & \\
\hline Grade 4 , excellent & $66(42.0)$ & $66(33.3)$ & $117(52.7)$ & \\
\hline Onset-to-selection time interval (hr) & $154(105-305)$ & $191(112-385)$ & $210(117-422)$ & 0.004 \\
\hline$<6$ & $121(77.1)$ & $138(69.7)$ & $144(64.9)$ & \\
\hline $6-8$ & $27(17.2)$ & $43(21.7)$ & $49(22.1)$ & \\
\hline $8-12$ & $9(5.7)$ & $17(8.6)$ & $29(13.1)$ & \\
\hline
\end{tabular}

Values are presented as number (\%), mean \pm standard deviation, or median (interquartile range).

NIHSS, National Institutes of Health Stroke Scale. 
Table 4. Multivariable analysis of the three selection methods

\begin{tabular}{|c|c|c|c|c|c|c|}
\hline \multirow{2}{*}{ Variable } & \multicolumn{2}{|c|}{ Clinical-core mismatch } & \multicolumn{2}{|c|}{ Target mismatch } & \multicolumn{2}{|c|}{ Collateral status } \\
\hline & OR $(95 \% \mathrm{Cl})$ & $P$ & OR (95\% Cl) & $P$ & OR $(95 \% \mathrm{Cl})$ & $P$ \\
\hline Initial NIHSS score & $1.52(1.38-1.68)$ & $<0.001$ & $1.04(0.98-1.11)$ & 0.200 & $0.96(0.92-1.02)$ & 0.179 \\
\hline Infarct core volume (mL) & $0.94(0.92-0.96)$ & $<0.001$ & $0.98(0.97-0.99)$ & $<0.001$ & $0.98(0.97-0.99)$ & $<0.001$ \\
\hline Penumbra/core volume ratio & & & $1.62(1.37-1.92)$ & $<0.001$ & $1.01(0.99-1.03)$ & 0.470 \\
\hline \multicolumn{7}{|l|}{ Collateral status } \\
\hline Grade 1, poor & Reference & & Reference & & $N A^{*}$ & \\
\hline Grade 2, intermediate & $0.58(0.17-1.96)$ & 0.383 & $0.90(0.32-2.55)$ & 0.838 & & \\
\hline Grade 3, good & $0.47(0.15-1.51)$ & 0.205 & $2.76(0.96-7.97)$ & 0.060 & & \\
\hline Grade 4, excellent & $0.82(0.25-2.71)$ & 0.748 & $0.34(0.11-1.04)$ & 0.060 & & \\
\hline Onset-to-selection time interval (min) & $0.995(0.993-0.998)$ & $<0.001$ & 0.998 (0.996-0.999) & 0.021 & & \\
\hline
\end{tabular}

$\mathrm{OR}$, odds ratio; $\mathrm{Cl}$, confidence interval; NIHSS, National Institutes of Health Stroke Scale; NA, not available.

${ }^{*}$ Collateral status could not be entered in the multivariable model owing to multicollinearity.

tial but supplementary information. Specifically, the clinicalcore mismatch selection method has an advantage in that it is a simple assessment method that does not require post-processing. However, this selection method may be highly dependent on the size of the infarct core and clinical features (e.g. infarct location). The target mismatch and collateral status selection methods are pathophysiologically plausible (penumbra and collaterals, respectively); however, they require post-processing. The target mismatch selection method has an advantage in that it provides clear visualization using a fast-automated software (RAPID software, iSchemaView, Stanford, CA, USA). The collateral status selection method is less dependent on the core size than the target mismatch selection method; the collateral status represents the perfusion status at later Tmax time points (capillary and venous phases) but not the extent of perfusion defects at earlier Tmax time points (arterial phase). ${ }^{11}$ In addition, the present study showed that the onsetto-selection time interval was not associated with collateral status profiles.

This study has certain limitations. First, this study was based on retrospective analyses of prospective cohorts from two university hospitals actively performing EVT. Therefore, the generalizability of the study findings may be limited. Second, this study cannot provide information on clinical outcomes after EVT according to the three different selection methods. In the DEFUSE-3 trial, pretreatment infarct core volumes of $<70 \mathrm{~mL}$ (too large for inclusion according to the DAWN trial criteria) are associated with benefits for EVT, and good leptomeningeal collaterals on single-phase $\mathrm{CT}$ angiography were not predictive of outcomes. ${ }^{12,13}$ Future studies are warranted to clarify this issue. Third, the clinical-core and target mismatch criteria were used to select patients among late-window patients in clinical trials, and relatively early-window patients were included in the two cohorts used in the present study. Although we used data from two university hospitals with different time interval criteria for the critical pathway for acute stroke to investigate different time periods, this study did not include patients who arrived after 12 hours of symptom onset; patients who presented to the hospitals before EVT at an extended time window were included. ${ }^{2,3}$ Therefore, further studies are needed to investigate the findings in patients arriving between 12 and 24 hours of symptom onset.

\section{Conclusions}

In conclusion, although the application of individual selection methods resulted in favorable outcomes after EVT in clinical trials, a significant number of patients who may have favorable responses to EVT could be missed depending on the selection method used. These selection methods may provide differential but supplementary information; certain key factors considered in one selection method do not influence the eligibility for EVT in other selection methods. Continuous efforts are needed to increase the number of patients who could undergo EVT.

\section{Supplementary materials}

Supplementary materials related to this article can be found online at https://doi.org/10.5853/jos.2019.01578.

\section{Disclosure}

The authors have no financial conflicts of interest. 


\section{Acknowledgments}

This study was supported by a grant from the National Research Foundation of Korea (No. 2018R1A2B2003489).

\section{References}

1. Saver JL, Goyal $M$, van der Lugt $A$, Menon $B K$, Majoie $C B$, Dippel DW, et al. Time to treatment with endovascular thrombectomy and outcomes from ischemic stroke: a metaanalysis. JAMA 2016;316:1279-1288.

2. Nogueira RG, Jadhav AP, Haussen DC, Bonafe $A$, Budzik RF, Bhuva $P$, et al. Thrombectomy 6 to 24 hours after stroke with a mismatch between deficit and infarct. N Engl J Med 2018; 378:11-21.

3. Albers GW, Marks MP, Kemp S, Christensen S, Tsai JP, OrtegaGutierrez S, et al. Thrombectomy for stroke at 6 to 16 hours with selection by perfusion imaging. $N$ Eng/ J Med 2018;378: 708-718.

4. Kim BM, Baek JH, Heo JH, Nam HS, Kim YD, Yoo J, et al. Collateral status affects the onset-to-reperfusion time window for good outcome. J Neurol Neurosurg Psychiatry 2018;89: 903-909.

5. Ribo $M$, Flores $A$, Rubiera $M$, Pagola J, Sargento-Freitas J, Rodriguez-Luna $D$, et al. Extending the time window for endovascular procedures according to collateral pial circulation. Stroke 2011;42:3465-3469.

6. Hwang YH, Kang DH, Kim YW, Kim YS, Park SP, Liebeskind DS. Impact of time-to-reperfusion on outcome in patients with poor collaterals. AJNR Am J Neuroradiol 2015;36:495500.

7. Galimanis A, Jung S, Mono ML, Fischer U, Findling O, Weck $A$, et al. Endovascular therapy of 623 patients with anterior circulation stroke. Stroke 2012;43:1052-1057.

8. Kim SJ, Son JP, Ryoo S, Lee MJ, Cha J, Kim KH, et al. A novel magnetic resonance imaging approach to collateral flow imaging in ischemic stroke. Ann Neurol 2014;76:356-369.

9. Kim JH, Bang OY, Liebeskind DS, Ovbiagele B, Kim GM, Chung CS, et al. Impact of baseline tissue status (diffusion-weighted imaging lesion) versus perfusion status (severity of hypoperfusion) on hemorrhagic transformation. Stroke 2010;41: e135e142.

10. Seners $P$, Roca $P$, Legrand $L$, Turc $G$, Cottier JP, Cho TH, et al. Better collaterals are independently associated with postthrombolysis recanalization before thrombectomy. Stroke 2019;50:867-872.

11. Son JP, Lee MJ, Kim SJ, Chung JW, Cha J, Kim GM, et al. Impact of slow blood filling via collaterals on infarct growth: comparison of mismatch and collateral status. J Stroke 2017; 19:88-96.

12. Leslie-Mazwi $T M$, Hamilton $S$, Mlynash $M$, Patel $A B$, Schwamm LH, Lansberg MG, et al. DEFUSE 3 non-DAWN patients. Stroke 2019;50:618-625.

13. de Havenon A, Mlynash M, Kim-Tenser MA, Lansberg MG, Leslie-Mazwi T, Christensen S, et al. Results from DEFUSE 3: good collaterals are associated with reduced ischemic core growth but not neurologic outcome. Stroke 2019;50:632638. 


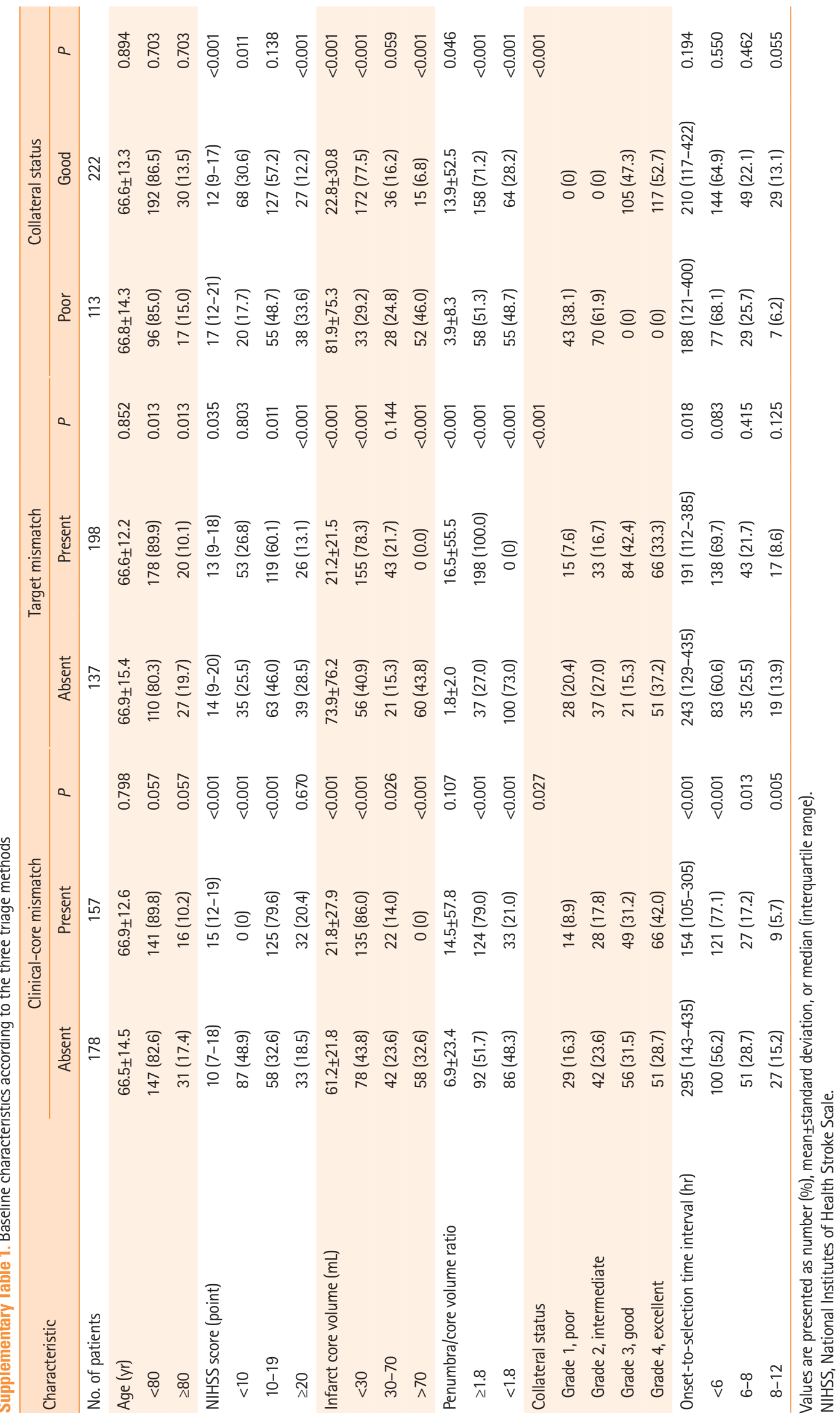


Clinical-core mismatch

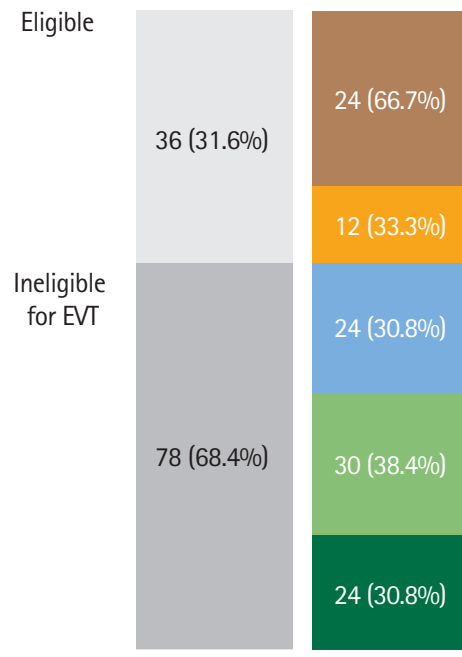

Target mismatch

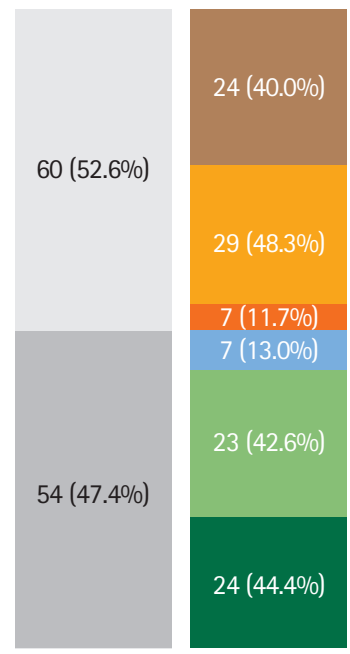

Collateral status

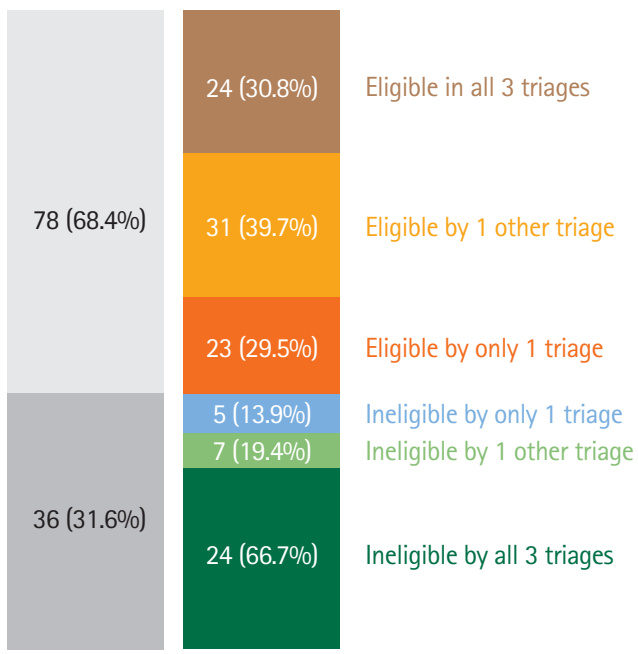

Supplementary Figure 1. Number of patients with favorable and unfavorable profiles at a later time period (onset-to-selection time interval, $>6$ hours) in the two cohorts according to the three selection methods. EVT, endovascular treatment. 